POPULATIONS

VUINERABLES 7|2021

Mobilité résidentielle et vulnérabilités

\title{
Introduction. Mobilité résidentielle et vulnérabilités
}

Introduction. Residential mobility and vulnerabilities

Céline Clément et Aurélien Dasré

\section{OpenEdition}

Journals

Édition électronique

URL : https://journals.openedition.org/popvuln/748

DOI : $10.4000 /$ popvuln.748

ISSN : 2650-7684

Éditeur

LIR3S - Laboratoire Interdisciplinaire de Recherche "Sociétés Sensibilités Soin" (UMR 7366 CNRS-uB)

Référence électronique

Céline Clément et Aurélien Dasré, «Introduction. Mobilité résidentielle et vulnérabilités », Populations vulnérables [En ligne], 7| 2021, mis en ligne le 01 décembre 2021, consulté le 26 février 2022. URL : http://journals.openedition.org/popvuln/748 ; DOI : https://doi.org/10.4000/popvuln.748

Ce document a été généré automatiquement le 26 février 2022.

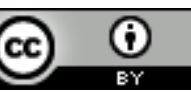

Les contenus de la revue Populations vulnérables sont mis à disposition selon les termes de la Licence Creative Commons Attribution 4.0 International 


\title{
Introduction. Mobilité résidentielle et vulnérabilités
}

\author{
Introduction. Residential mobility and vulnerabilities
}

Céline Clément et Aurélien Dasré

1 La plupart des recherches s'accordent pour estimer que la mobilité résidentielle n'est pas un simple déplacement. C'est un phénomène complexe qui interagit avec d'autres (C. Bonvalet et J. Brun, 2003). Elle apparait alors comme une expérience sociale totale (V. Kaufmann, 2005), impliquant de tenir compte de l'ensemble des sphères de la vie et de leur intrication pour l'analyser, ces dernières s'influençant mutuellement. Ainsi, l'entrée à l'université, la mise en couple, une naissance, un changement d'emploi, la retraite sont autant d'évènements susceptibles d'entraîner un déménagement et un changement de statut résidentiel. Inversement, une mobilité résidentielle peut conduire à une rupture amoureuse, à s'éloigner de son réseau familial et amical, ou à une reconversion professionnelle. Les trajectoires s'entrelacent donc, se coconstruisent, et "les formes de mobilité - sociale, géographique, familiale - "ne [seraient] en réalité que les diverses facettes d'un processus unique de réaménagement de l'existence" (Y. Grafmeyer, 1994)» (C. Bonvalet et J. Brun, 2003).

2 Ces mêmes recherches ont également mis en lumière la diversité et la complexité du déroulement des trajectoires, autrefois lues comme linéaires et relativement homogènes. Or, du fait de parcours de vie familiaux et professionnels plus flexibles, d'un nouveau code biographique mettant « l'accent sur le développement de l'individu et la possibilité (et nécessité) de faire des choix» (M. Kholi, 1989), les parcours résidentiels sont devenus moins prévisibles, parfois réversibles. Ce mouvement d'individualisation des parcours de vie peut s'accompagner d'une précarisation des trajectoires résidentielles conduisant à un « déclassement " (résidentiel et social, les deux étant fréquemment liés), et ce, à différents moments de l'existence. Certains événements, comme la séparation, peuvent aussi induire (théoriquement) une mobilité d'au moins un des deux conjoints ${ }^{1}$, quand des difficultés de santé, financières, la perte d'un conjoint, peuvent amener de véritables bifurcations (C. Bidart, 2006 ; M. Grossetti, M. Bessin et C. Bidart, 2009). 
3 Ce numéro de la revue Populations vulnérables invite à interroger les trajectoires résidentielles associées aux parcours de vie et les évènements qui viennent les infléchir, voire les déstabiliser. Il rassemble des articles portant sur l'ensemble des âges de la vie, allant de l'entrée dans la vie adulte (Sami Zegnani, Frédérique Quidu et Alexandra Filhon) à la vieillesse (Manon Labarchède; Armelle Klein et Frédéric Sandron) et s'attache à des sous-populations spécifiques, comme les familles sans logement en Île-de-France (Candy Jangal) et à plusieurs évènements - la séparation (Laure Crépin), la maladie (Manon Labarchède) - venant impliquer un réagencement des parcours résidentiels du fait des effets de contamination d'une sphère de vie à l'autre (C. Bidart, 2006).

4 S'il existe une hétérogénéité dans l'enchainement, le nombre et la nature des différentes trajectoires résidentielles analysées dans ce numéro, on peut dégager de ces cinq articles quelques traits communs. Ils décrivent l'enchainement de parcours résidentiels, dans une perspective le plus souvent longitudinale, et s'appuient sur des données quantitatives (en particulier des enquêtes de l'Insee, avec l'enquête SRCV ou EVA) et/ou qualitatives (récits de vie, récits de vie spatialisés) afin d'en saisir la dynamique et l'évolution.

5 Plusieurs d'entre eux montrent l'importance de tenir compte de l'entourage (C. Bonvalet et É. Lelièvre, 2012), qu'il soit familial ou institutionnel (Candy Jangal), pour mieux appréhender les mobilités résidentielles. La famille fournit une clef de compréhension à la mobilité - ou à l'immobilité - des individus. Elle peut expliquer un déplacement ou au contraire un ancrage résidentiel (C. Vignal, 2014 ; T. Venet, 2017), comme l'indique l'article d'Armelle Klein et de Frédéric Sandron qui porte sur les personnes âgées à la Réunion. Elle peut aussi constituer une aide matérielle favorisant la mobilité, en particulier au moment de la décohabitation adulte ${ }^{2}$, ou au contraire l'immobilité, en prolongeant la période de cohabitation, forme de "cohabitation négociée » (C. Van de Velde, 2008) difficilement vécue par les jeunes adultes comme le souligne l'article de Sami Zegnani, Frédérique Quidu et Alexandra Filhon. À l'autre extrémité de la vie, la famille peut encore représenter une ressource, notamment lorsque les enfants devenus adultes offrent une "protection rapprochée " à leurs parents vieillissants et malades (Manon Labarchède). Finalement, ces deux articles, l'un sur l'entrée dans la vie adulte, l'autre sur l'entrée en établissement des personnes atteintes d'une maladie d'Alzheimer ou d'une maladie apparentée révèlent une inversion du soutien familial, où les enfants deviennent «aidants", mais aussi, en creux, des représentations de la mobilité. Alors qu'il s'agit d'acquérir son autonomie par le biais de la décohabitation (être mobile), il convient de la conserver en restant le plus longtemps possible dans son logement (rester immobile). Cela nuance alors la valorisation positive de la mobilité, tout au moins aux différents moments des parcours de vie, l'injonction à la mobilité lue comme construction de l'autonomie s'inversant au grand âge. Plus généralement, les articles relèvent des rapports sociaux qui traversent la mobilité : rapports sociaux d'âge mais aussi de classe, les jeunes ne disposant pas des mêmes ressources matérielles et symboliques pour décohabiter (Sami Zegnani, Frédérique Quidu et Alexandra Filhon); rapports sociaux de sexe comme le montre Laure Crépin qui explore les inégalités genrées d'accession à la propriété suite à une séparation conjugale. Enfin, ils témoignent, en creux, de l'insuffisance des politiques publiques à chaque âge de la vie : lors de l'enfance (Candy Jangal), de la jeunesse (Sami Zegnani, Frédérique Quidu et Alexandra Filhon), au cours de la vieillesse (Armelle Klein 
et Frédéric Sandron ; Manon Labarchède), ou encore au moment de l'âge de la maturité (Laure Crépin).

6 Les articles ont été regroupés sous deux thématiques entrelacées. Les trois premiers articles épousent la logique des parcours de vie en privilégiant deux moments-clefs : l'entrée dans la vie adulte (Sami Zegnani, Frédérique Quidu et Alexandra Filhon) et dans la vieillesse (Armelle Klein et Frédéric Sandron ; Manon Labarchède).

7 La décohabitation parentale constitue un moment clef des trajectoires résidentielles, ainsi qu'un moment fort des transitions biographiques. Analysée plus spécifiquement à partir des années 1980, lorsque les sociologues constatent un allongement de la jeunesse, concluant alors à un nouvel «âge de la vie» (0. Galland, 1991), plusieurs recherches ont depuis relevé une hétérogénéité sociale des trajectoires de décohabitation des jeunes, certains apparaissant vulnérables (P.Cordazzo, 2018), parfois «soumis" à des retours au domicile parental - nommés «enfants boomerang » (B. Mitchell, 2005) -, quand d'autres sont « contraints » à des immobilités (T. Venet, 2017).

8 Dans leur article "Vulnérabilités et trajectoires résidentielles des jeunes adultes en France ", Sami Zegnani, Frédérique Quidu et Alexandra Filhon reviennent sur cette étape constitutive de l'entrée dans la vie adulte. S'appuyant sur l'enquête EVA de l'Insee, il et elles proposent une approche longitudinale, croisant vulnérabilité objective (origine sociale, sexe, diplôme) et vulnérabilité ressentie par les jeunes. Les auteur.e.s étudient ensuite les liens entre ces vulnérabilités et les trajectoires résidentielles à partir de la décohabitation parentale. Trois trajectoires types et deux extrêmes sont présentées. Les jeunes quittant le domicile parental pour louer un logement en ne faisant pas appel à l'aide financière des parents (les indépendants); les départs précoces financièrement aidés; les "cohabitants ", qui restent tardivement chez leurs parents. Entre ces trois trajectoires, les sentiments de vulnérabilité sont partagés, bien que les cohabitants soient plus vulnérables que les indépendants. Les deux dernières trajectoires sont plus marquées, avec les "primoaccédants » qui apparaissent particulièrement optimistes, et les « instables », qui sont les plus vulnérables. Finalement, on peut noter combien la norme de l'indépendance résidentielle reste forte: c'est parmi ceux qui ont le plus de difficulté à quitter le domicile de leurs parents que le sentiment de vulnérabilité est le plus aigu. Les situations où le domicile familial offre soutien relationnel et matériel des parents (partage d'un logement, de repas) ne semblent donc pas sécuriser pleinement les trajectoires d'entrée dans la vie adulte, en particulier des jeunes des classes populaires qui peuvent le vivre comme un échec (E. Maunaye et al., 2019). Plus généralement, les aides financières des parents doivent demeurer temporaires, ces dernières engendrant un sentiment de fragilité lorsqu'elles prolongent la période de dépendance vis-à-vis de la famille d'origine. Cela questionne dès lors les politiques publiques mises en place à l'égard des jeunes en France, qui les placent bien souvent sous la protection de leur famille ${ }^{3}$.

9 Tout comme la jeunesse, la vieillesse a connu de profondes transformations et est aujourd'hui appréhendée comme un processus long, graduel et réversible (C. Clément et $\mathrm{C}$. Brugeilles, 2020), dont les conditions varient fortement selon le milieu social et le sexe. Dans ce cheminement, les individus peuvent être amenés, selon les événements ou les épreuves de la vie, à redéfinir leurs « choix » résidentiels (Y. Grafmeyer, 2010) en fonction des contextes territoriaux, mais aussi des logiques familiales et de santé. 
10 Dans leur article, Armelle Klein et Frédéric Sandron abordent la relation entre mobilité résidentielle et vulnérabilité des personnes âgées dans l'environnement spécifique de l'île de la Réunion, caractérisée par des conditions de vie - et de santé plus précaires qu'en métropole ainsi que par des vulnérabilités géophysiques.

11 En se basant sur un corpus de quatorze entretiens semi-directifs réalisés auprès de personnes ayant récemment effectué une mobilité résidentielle à la Réunion, les auteur.e.s recherchent les facteurs de vulnérabilité ayant entrainé une mobilité ou induisant un désir de mobilité. Ils en relèvent quatre, tout en soulignant qu'un même facteur peut engendrer ou, au contraire, entraver une mobilité souhaitée. Un premier facteur de vulnérabilité, de nature économique, peut inciter à la mobilité (recherche d'un cadre de vie moins onéreux), comme l'empêcher, du fait de son coût. Des facteurs liés à la santé, en particulier mentale et psychologique, influent également sur les décisions de mobilité. Le manque de relations sociales et familiales, qui peut s'avérer douloureux pour des personnes venues s'installer à la Réunion une fois à la retraite, constitue aussi un facteur de vulnérabilité. Enfin, l'environnement domestique et territorial, notamment en lien avec la topographie de la Réunion, rend les déplacements particulièrement délicats sur l'île. Les auteur.e.s montrent alors que la mobilité est rarement anticipée et intervient souvent lorsque plusieurs de ces facteurs se cumulent et ne peuvent plus être compensés par des aménagements matériels et/ou l'intervention d'aidants. Le climat, la topographie et l'insularité de la Réunion rendent ces vulnérabilités particulièrement prégnantes. Les auteur.e.s soulignent ainsi la nécessité de « l'adaptation locale de la société au vieillissement de la population ». établissement des personnes âgées atteintes d'une maladie neurodégénérative. Elle propose d'interroger « la capacité de cette population vulnérable à anticiper et à coconstruire avec leurs aidant.e.s l'entrée en établissement pour en faire une mobilité résidentielle choisie ». La nature évolutive de ces maladies induit un temps parfois relativement long entre le diagnostic et la nécessaire entrée en institution spécialisée, d'autant que plusieurs stratégies sont mises en place pour retarder cette entrée. Ce temps est diversement mis à profit pour préparer l'entrée en institution. Dans les cas où la mobilité vers l'institution n'est pas anticipée (quelquefois par déni de la maladie), le placement se fait dans la précipitation et repose uniquement sur les aidant.e.s, sans laisser de place à une période de transition. Au contraire, l'anticipation de l'évolution de la maladie et donc de la nécessité de trouver un lieu d'accueil adapté permet des transitions plus douces, où la personne malade peut infléchir le lieu de la mobilité résidentielle. L'auteure montre comment cette " dernière » mobilité se co-construit et s'articule, selon la trajectoire résidentielle du malade, de la maladie, mais aussi de l'aide et de l'aidant.e. En particulier, dans une logique de maintien à domicile, logique qui répond à la norme de l'autonomie et qui émane tant des parents que des enfants, mais aussi de l'État qui privilégie l'aide à la personne, elle rappelle les difficultés auxquelles les aidant.e.s doivent faire face, allant parfois jusqu'à l'épuisement, alors même qu'ils font partie des catégories les moins fragiles. Elle dévoile également l'absence d'accompagnement des aidant.e.s dans leur recherche de solution pour leur proche lorsque le maintien à domicile devient problématique.

13 Les deux articles suivants ont pour élément commun de se situer au moment de l'âge adulte. Ils analysent la mobilité au prisme d'événements "déstabilisateurs»: la séparation, la perte d'un logement, une migration internationale. Ils portent plus 
particulièrement sur l'intrication des trajectoires, notamment résidentielle et conjugale (Laure Crépin), ou encore de santé (Candy Jangal).

La mobilité résidentielle dépend fortement des événements familiaux: la mise en couple, l'arrivée des enfants (C. Kersuzan, 2009), une séparation (S. Villaume, 2016), une recomposition familiale peuvent conduire à un déménagement et à un changement de statut résidentiel. Plusieurs recherches se sont penchées sur les effets d'un divorce (P.Festy, 1990) ou d'une recomposition familiale (D. Le Gall et C.Martin, 1991; C. Clément et C. Bonvalet, 2006), sur les trajectoires résidentielles, démontrant une plus grande mobilité des hommes et des femmes divorcés, un changement du type de logement et la perte du statut de propriétaire, en particulier pour les femmes (C. Bonvalet et A. Bringé, 2016), alors même que celui-ci constitue bien souvent un gage de sécurité4. Ces recherches ont aussi souligné des effets liés au genre et à la garde des enfants: les hommes qui ont la garde des enfants, sont davantage sédentaires que les femmes, quelles que soient les étapes considérées, l'après rupture ou la recomposition familiale.

Laure Crepin prolonge ces recherches dans son article en proposant de traiter les inégalités de genre en matière de propriété du logement et leur évolution suite à une rupture conjugale. En mobilisant les données longitudinales de l'enquête SRCV de l'Insee, elle démontre comment les inégalités d'activité professionnelle antérieures à la séparation se transforment en inégalités de maintien ou d'accès à la propriété après la séparation. En moyenne, les hommes restent plus souvent que les femmes dans le logement acheté au sein du couple et sont, globalement, plus souvent propriétaires que leur ex-conjointe deux ans après la séparation. Cette inégalité s'accroit dans les classes moyennes et populaires, mais également lorsque des enfants sont encore à charge au moment de la séparation: les hommes sont davantage propriétaires lorsqu'ils ont la garde de leurs enfants. Si le cumul des difficultés féminines issues de leur trajectoire professionnelle (positions professionnelles inférieures, carrières discontinues, niveaux de vie plus faibles) explique en partie ces trajectoires résidentielles genrées, Laure Crépin les relie aux représentations de la maternité et de la paternité. Il n'en reste pas moins que ces trajectoires résidentielles genrées viennent redoubler les inégalités existantes, la propriété constituant bien souvent un gage de sécurité.

$\mathrm{Si}$ les interactions entre trajectoires résidentielle et professionnelle, mais aussi familiale, ont été particulièrement étudiées, celles avec les trajectoires de santé sont plus rares. C'est ce qu'expose Candy Jangal dans son article « Comprendre l'impact du parcours résidentiel sur le suivi de soins de l'enfant sans logement » qui présente une étude du lien entre les parcours résidentiels instables des familles sans domicile fixe et le suivi de soins des enfants âgés de moins de six ans. L'originalité tient à la méthodologie utilisée, laquelle s'appuie sur dix-huit récits de vie spatialisés permettant d'illustrer à la fois les ruptures de parcours résidentiels et les ruptures ou reprises de soins des enfants. Elle montre dans un premier temps le rôle du type d'hébergement dans l'entrée dans les parcours de soins mais aussi que les mobilités résidentielles de ces populations sont très fréquentes (2,2 déménagements par an en moyenne) et impliquent parfois des changements de commune voire de département. Ces itinéraires résidentiels morcelés rendent très complexe les suivis de santé des enfants présentant des maladies chroniques. Les déménagements nécessitent en effet de changer régulièrement de structures de soins, ce qui allonge considérablement la prise en charge des enfants, montrant combien le changement de contexte spatial est capital et 
que l'on ne peut pas raisonner qu'en terme de logement. Pour autant, les liens ne sont pas mécaniques : un déménagement n'entraine pas systématiquement une rupture ou un changement de professionnels de santé quand le contexte institutionnel façonne ces parcours de soins.

\section{BIBLIOGRAPHIE}

Bonvalet C. et Brun J. (2002), «État des lieux des recherches sur la mobilité résidentielle en France ", in Lévy J.-P. et Dureau F. (dir.), L'accès à la ville, les mobilités spatiales en question, Paris, L'Harmattan, p. 15-64.

Bonvalet C. et Lelièvre É. (2012), De la famille à l'entourage, Paris, INED.

Bidart C. (2006), « Crises, décisions et temporalités : autour des bifurcations biographiques », Cahiers internationaux de sociologie, vol. CXX, p. 29-57.

Bonvalet C. et Bringé A. (2016), «

Charles N., Portela M. et Raynaud É. (coord.) (2019), « Un regard renouvelé sur les ressources des jeunes : ressources matérielles, soutien, accès aux capacités ", Revue française des affaires sociales, $\mathrm{n}^{\circ} 2$, p. 5-21.

Clément C. et Brugeilles C. (2020), Introduction à la démographie, Paris, Armand Colin.

Clément C.et Bonvalet C. (2006), Les espaces de vie des familles recomposées, Paris, PUCA, $\mathrm{n}^{\circ} 161$, $138 \mathrm{p}$.

Cordazzo P. (dir.) (2019), « Parcours d'étudiants. Sources, enjeux et perspectives de recherche », Paris, Ined, $240 \mathrm{p}$.

Festy P. (1990), « Mobilité résidentielle des femmes séparées : une étape dans le cycle familial », in Bonvalet C. et Fribourg A. M. (dir.), Stratégies résidentielles, Paris, INED, p. 231-263.

Galland O. (1991), Sociologie de la jeunesse, Paris, Armand Colin.

Grafmeyer Y. (2010), « Approches sociologiques des choix résidentiels », in Authier J.-Y., Bonvalet C. et Lévy J.-P., Élire domicile. La construction sociale des choix résidentiels, Lyon, Presses universitaires de Lyon.

Grossetti M., Bessin M. et Bidart C. (2009), Les sciences sociales face aux ruptures et à l'événement, Paris, La Découverte.

Kaufmann V. (2005), « Mobilités et réversibilités : vers des sociétés plus fluides ? , Cahiers internationaux de sociologie, vol. 118, $\mathrm{n}^{\circ} 1$, p. 119-135.

Kersuzan C. (2009), « Changement de logement et naissance des enfants », Recherches familiales, $n^{\circ} 6$, p. 7-25.

Kohli M. (1989), « Le cours de vie comme institution sociale », Enquête, nº 5.

Le Gall D. et Martin C. (1991), Recomposition familiale et usage de l'espace domestique, Paris, Plan Construction et Architecture. 
Martin C., Cherlin A. et Cross-Barnet C. (2011), « Living Together Apart: Vivre ensemble séparés. Une comparaison France-États-Unis », Population, vol. 66, n os3-4, p. 647-669.

Maunaye E. et al. (2019), « Le domicile familial comme ressource ? Expériences de recohabitation dans les transitions vers l'âge adulte », Revue française des affaires sociales, n² 2, p. 143-166.

Mitchell B. (2006), The Boomerang Age. Transitions to Adulthood in Families, London, Adline Transaction.

Rault W. et Régnier-Loilier A. (2020), « Continuer à vivre sous le même toit après la séparation », Population \& Sociétés, vol. 582, n 10.

Van De Velde C. (2008), Devenir adulte. Sociologie comparée de la jeunesse en Europe, Paris, PUF.

Venet T. (2017), « Mobilité, ancrage et rapport à l'espace des jeunes des classes populaires rurales ", Savoir/Agir, vol. 39, $\mathrm{n}^{\circ}$ 1, p. 42-48.

Vignal C. (2014), «L'ancrage local, une ressource pour les classes populaires des territoires désindustrialisés », in Fol S., Miot Y. et Vignal C. (dir.), Mobilités résidentielles, territoires et politiques publiques, Villeneuve d'Ascq, Presses universitaires du Septentrion.

Villaume S. (2016), « Naissances, séparations et changements de logement », Revue française des affaires sociales, $\mathrm{n}^{\circ} 3$, p. 29-64.

\section{NOTES}

1. Il arrive que des couples qui se considèrent séparés continuent à vivre dans le même logement, phénomène nommé LTA (Living Together Apart) et analysé par C. Martin, A. Cherlin et C. CrossBarnet (2011). Ces situations semblent répondre au souhait de maintenir des liens avec des enfants communs, à des difficultés économiques engendrées par une éventuelle "rupture résidentielle » ou encore à l'espoir d'un retour à une vie conjugale «normale ». Si ces situations restent marginales, W. Rault et A. Régnier Loilier (2020) rappellent que, tout comme la mise en couple, la rupture est un processus. Un certain nombre d'ex-conjoints cohabitent donc ensemble pour une durée plus ou moins longue après avoir décidé de se séparer, notamment lorsqu'ils ont des enfants de moins de 15 ans.

2. Ce que Cécile Van de Velde (2008) nomme «l'extension du nid ».

3. Voir plus particulièrement le numéro de RFAS coordonné par Nicolas Charles et al., "Un regard renouvelé sur les ressources des jeunes: ressources matérielles, soutien, accès aux capacités ", $\mathrm{n}^{\circ} 2,2019$.

4. En revanche, les femmes obtiennent plus fréquemment un logement social que les hommes

(C. Bonvalet et A. Bringé, 2016).

\section{INDEX}

Mots-clés : vulnérabilités, mobilités résidentielles, parcours de vie

Keywords : vulnerabilities, residential mobility, life course 
AUTEURS

CÉLINE CLÉMENT

Maitresse de conférences en démographie

Université Paris Nanterre, CRESPPA-UMR 7217

AURÉLIEN DASRÉ

Maitre de conférences en démographie

Université Paris Nanterre, CRESPPA-UMR 7217 